\title{
Heterostructure InGaAIAs/InAIAs on the InP substrate for the electro-optical modulator based on the quantum confined Stark effect
}

\author{
Dmitry Gulyaev ${ }^{1, *}$, Dmitry Dmitriev ${ }^{1}$, Alexander Toropov ${ }^{1}$, Nataly Valisheva ${ }^{1}$, \\ Andrey Tsarev ${ }^{1}$, Eugeny Kolosovsky ${ }^{1}$, Leonid Feduchin ${ }^{1}$, Alexander Gorchakov ${ }^{2}$, \\ and Konstantin Zhuravlev ${ }^{1,2}$ \\ ${ }^{1}$ Rzhanov Institute of Semiconductor Physics, Lavrentiev av. 13, Novosibirsk, 630090, Russia \\ ${ }^{2}$ Novosibirsk State University, st. Pirogova 2, Novosibirsk, 630090, Russia
}

\begin{abstract}
The energy structure and the value of the electrooptic effect in heteroepitaxial structures (HES) with multiple InGaAlAs / InAlAs quantum wells have been studied. The interferometric method has been developed to determine small changes in the refractive index under transverse transmission of light through a layered structure. The length of the Mach-Zehnder interferometer for the modulator at HES with multiple InGaAlAs / InAlAs quantum wells has been chosen.
\end{abstract}

\section{Introduction}

Electrooptical modulators are one of the basic elements to construct devices for radiophotonics. Among many material platforms used to create such electro-optical modulators as lithium niobate [1], polymers [2], silicon-on-insulator [3], the modulators based on the quantum-dimensional Stark effect in structures with the multiple quantum wells epitaxially grown on InP substrates are very promising [4]. This is primarily due to the possibility of creating a completely monolithic integrated radiophotonic circuit on the basis of such a platform [5].

Therefore, the tasks aimed at optimizing the design of a heterostructure (HES) with multiple quantum wells on the InP have not lost their relevance or practical significance yet. The active waveguide region of such a structure should, on the one hand, have a large electro-optical effect for modulating the optical signal, and on the other hand, low absorption for wave propagation along the waveguides. Recently, we have proposed a HES in a double-comb waveguide based on the four-component InGaAlAs / InAlAs multiple quantum wells as a HES for a modulator [6].

In this work, we have determined a typical value of the electro-optical effect in such multiple quantum wells and estimated the required length of the Mach-Zehnder interferometer to construct a modulator on a heterostructure with InGaAlAs/InAlAs multiple quantum wells.

*Corresponding author: gulyaev@,isp.nsc.ru 


\section{Experimental methods}

Test heterostructures on the InP substrates were grown by molecular beam epitaxy to determine the magnitude of the electro-optical effect in multiple InGaAlAs/InAlAs quantum wells. The growth conditions for such structures are described in the article [7]. The design of the test HES is presented in Table 1. The thickness of the $\operatorname{In}_{0.52} \mathrm{Al}_{0.1} \mathrm{Ga}_{0.38} \mathrm{As}$ and $\mathrm{In}_{0.52} \mathrm{Al}_{0.48} \mathrm{As}$ layers of the multiple quantum wells was 17 and $10 \mathrm{~nm}$, respectively.

Table 1. Design of test heterostructure.

\begin{tabular}{|c|c|c|c|c|c|}
\hline No & Alloy & $\begin{array}{c}\text { Doping } \\
\text { type }\end{array}$ & $\begin{array}{l}\text { Doping } \\
\text { level, } \mathbf{c m}^{-3} \\
\end{array}$ & $\begin{array}{c}\text { Thickness, } \\
\text { nm }\end{array}$ & $\mathbf{n}$ \\
\hline 1 & $\mathrm{In}_{0.53} \mathrm{Ga}_{0.47} \mathrm{As}$ & $\mathrm{p}$ & $10^{19}$ & 100 & $3.5129+0.2318 \mathrm{i}$ \\
\hline 2 & $\mathrm{In}_{0.52} \mathrm{Al}_{0.48} \mathrm{As}$ & $\mathrm{p}$ & $10^{18}$ & 200 & $3.2097+0.008 \mathrm{i}$ \\
\hline 3 & $\mathrm{In}_{0.52} \mathrm{Al}_{0.48} \mathrm{As}$ & $\mathrm{p}$ & $510^{16}$ & 200 & $3.2097+0.001 \mathrm{i}$ \\
\hline 4 & $\begin{array}{c}\mathrm{In}_{0.52} \mathrm{Al}_{0,1} \mathrm{Ga}_{0.38} \mathrm{As} \\
/ \mathrm{In}_{0.52} \mathrm{Al}_{0.48} \mathrm{As} \text { quantum } \\
\text { well* } \\
\end{array}$ & $\mathrm{i}$ & - & 300 & 3.3185 \\
\hline 5 & $\mathrm{In}_{0.52} \mathrm{Al}_{0.48} \mathrm{As}$ & $\mathrm{n}$ & $510^{16}$ & 200 & $3.2020+0.0005 \mathrm{i}$ \\
\hline 6 & $\mathrm{In}_{0.52} \mathrm{Al}_{0.48} \mathrm{As}$ & $\mathrm{n}$ & $510^{17}$ & 200 & $3.1800+0.004 i$ \\
\hline 7 & $\mathrm{In}_{0.52} \mathrm{Al}_{0.48} \mathrm{As}$ & $\mathrm{n}$ & $510^{18}$ & 200 & $3.2026+0.1226 \mathrm{i}$ \\
\hline 8 & $\operatorname{InP}$ & SI & - & $362 \mathrm{mkm}$ & 3.17 \\
\hline
\end{tabular}

The energy spectrum of such quantum wells was determined from the photoluminescence (PL) spectra. The PL was excited by HeNe laser with a power of $5 \mathrm{~mW}$, and the PL was recorded by a Ge detector.

To determine the electro-optical effect in the test structure the mesa structures were etched, which allowed applying the voltage between the upper contact layer (the upper electrode in the form of a ring) and the lower contact layer. It is rather difficult to directly determine the value of the electro-optical effect from measuring the reflection coefficient of such a HES due to the small thickness of the multiple quantum wells compared to the total thickness of the heterostructure. Therefore, the measurement of the electro-optical effect in the HES was carried out by the IR-interferometric technique. Although the layer is thin, namely, $300 \mathrm{~nm}$, multiple interference of light in it allows registering relatively small changes in the refractive index. Moreover, reflection and transmission of the p-polarized light that is more sensitive to changes in the refractive index of the surface layers is used to increase the sensitivity of the method. For this purpose, a Glan prism is installed on the optical way to the sample (see Fig. 1). The test sample itself is located on a goniometric base that provides rotation around the vertical axis and allows measuring the angular dependence of the reflection/transmission coefficient with an accuracy of 1 min. Sample positioning is provided with micron accuracy. The registration of the reflected beam was carried out by the upper electrode in the form of a ring by the Ge photodetector. The pulse applied voltage is used to reduce the effect of sample heating. In this case, the application of the electric field to the structure and the measurement of the reflected/transmitted infrared radiation are synchronized. 


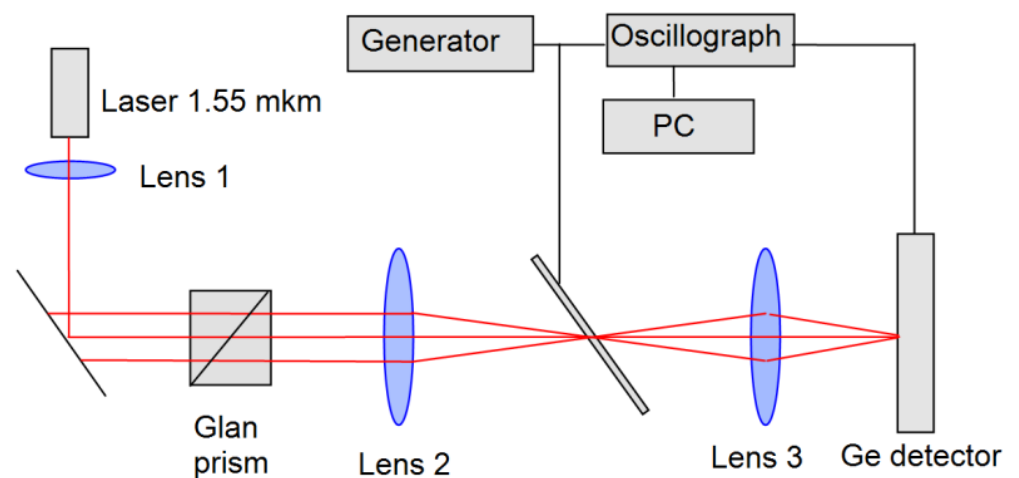

Fig.1. The transmission setup for measuring the electro optic effect in the HES.

The following mathematical model was used to interpret the interference pattern. Let an optical wave with a flat front fall on the layered structure (see Fig. 2).

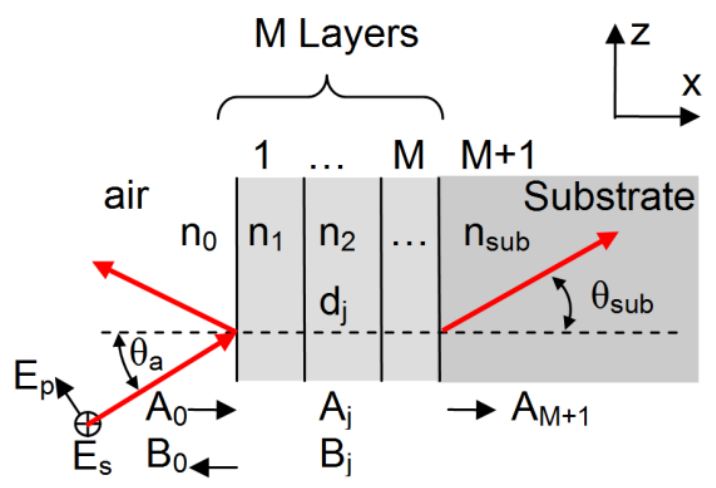

Fig. 2. Light incident with $p$ (TM) or $s$ (TE) polarization on a layered structure with the $\mathrm{M}$ layers $\{\mathrm{dj}, \mathrm{nj}\}$.

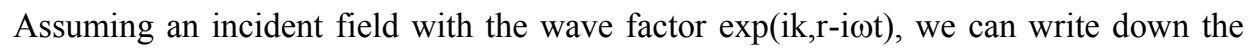
transverse field distribution ( $\psi=$ Ey or $\psi=$ Hy for TE or TM polarization, respectively) in every layer in the form of a superposition of the direct $(\mathrm{Aj})$ and reverse $(\mathrm{Bj})$ waves as:

$$
\left\{\begin{array}{c}
\psi=A_{j} \exp \left(i k_{j}\left(x-x_{j}\right)\right)+B_{j} \exp \left(i k_{j}\left(x_{j+1}-x\right)\right) \\
\psi^{\prime}=i k_{j}\left[A_{j} \exp \left(i k_{j}\left(x-x_{j}\right)\right)-B_{j} \exp \left(i k_{j}\left(x_{j+1}-x\right)\right)\right.
\end{array}\right.
$$

where $\kappa_{\mathrm{j}}=k_{0} n_{j} \cos \left(\theta_{j}\right)$ is the transverse (with respect to the layers) wave number in the $\mathrm{jth}$ layer, and $k_{0}=2 \pi / \lambda_{0}$ is the wave number of light in vacuum. [8] In the ellipsometric terminology [8] TE/TM polarizations correspond to the $E s$ and $E p$ waves in Fig1. Using (1) and applying consistently the boundary continuity conditions on vertical interfaces, one can express the amplitude coefficients inside every layer through the amplitudes $A_{0}, B_{0}$ of the incident and reflected waves in the air as:

$$
\left[\begin{array}{c}
A_{j+1} \\
B_{j+1}
\end{array}\right]=G_{j} E_{j} \ldots G_{1} E_{1} G_{0}\left[\begin{array}{c}
A_{0} \\
B_{0}
\end{array}\right]
$$

where $G j=\frac{1}{2}\left[\begin{array}{cc}1+g_{j} & 1-g_{j} \\ 1-g_{j} & 1+g_{j}\end{array}\right], \quad E j=\left[\begin{array}{cc}e^{i k_{j} d_{j}} & 0 \\ 0 & e^{-i k_{j} d_{j}}\end{array}\right], g_{j}=\kappa_{j} / \kappa_{j+1}$ for the TE polarization and $g_{j}=\left(\kappa_{j} / \kappa_{j+1}\right) \cdot\left(n_{j+1} / n_{j}\right)^{2}$ for the TM polarization of the incident light. As you 
can see, the matrixes $G_{j}$ and $E_{j}$ have a small dimension of $2 \times 2$, therefore, for the total number of layers (for $j=\mathrm{M}$ ) matrix multiplication gives the resulting matrix $\mathrm{U}$ also of dimension $2 \times 2$, resulting in only four a $u_{i k}$ :

$$
\left[\begin{array}{l}
A_{M+1} \\
B_{M+1}
\end{array}\right]=U\left[\begin{array}{l}
A_{0} \\
B_{0}
\end{array}\right]
$$

The expression (3) is basic in solving the problem of the light transmission through a multilayer structure. For a substrate of unlimited thickness, the backward wave is absent and $B_{M+1}=0$. Therefore:

$$
\left[\begin{array}{ll}
u_{11} & u_{12} \\
u_{21} & u_{22}
\end{array}\right]\left[\begin{array}{l}
A_{0} \\
B_{0}
\end{array}\right]=\left[\begin{array}{c}
A_{M+1} \\
0
\end{array}\right]
$$

whence we can express the complex R-reflection and T-transmission coefficients explicitly:

$$
B_{0}=-\frac{u_{21}}{u_{22}} \cdot A_{0}=R \cdot A_{0} ; A_{M+1}=\frac{u_{11} u_{22}-u_{12} u_{21}}{u_{22}} \cdot A_{0}=T \cdot A_{0}
$$

In case of finite thickness, the substrate serves as the Mth layer, the light goes out into the continuous air half-space and the equations $(4,5)$ are still satisfied.

A solution in the form of (1) satisfies the wave equation and boundary conditions and represents the so-called radiative mode of the continuous spectrum with respect to the incident angle $\theta_{a}$ [8]. To resolve a localized waveguide mode with a discrete (longitudinal) wave number $\beta_{m}$, it is necessary to include an additional condition in (3): $A_{0}=0, B_{M+1}=0$, which gives $A_{M+1}=u_{12} B_{0}$ and $u_{22} B_{0}=0$. Thus, $u_{22}\left(\beta_{m}\right)=0$ is the dispersion equation for the mode solution:

$$
\psi(x, z, t)=\psi(x) \cdot \exp \left(i \beta_{m}-i \omega t\right)
$$

The expressions (3-6) are valid for absorbing layers with complex permittivity. The choice of the sign of the imaginary part of the dielectric constant $\varepsilon_{j}=\varepsilon^{\prime}+i \varepsilon^{\prime \prime}, \varepsilon^{\prime \prime}>0$ (in our case, positive) corresponds to the field decay during the propagation due to absorption.

An effective solution for R/T coefficients can be obtained in a slightly more complicated way, using the Fresnel's formulas and the stable Scandonne-Ballerini's recurrence algorithm [9]. In this case, our results were identical with (5).

\section{Results and discussion}

Figure 3 shows the PL spectrum of the multiple quantum well. As one can see from the figure, the PL peak of the multiple QWs is near $1.4 \mu \mathrm{m}$, which is $150 \mathrm{~nm}$ less than the modulator operating wavelength of $1.55 \mu \mathrm{m}$ and guarantees low absorption of the light wave propagating through the waveguide (layer of the MQW).

Figure 4 shows the typical experimental and calculated (based on the sum of plane waves, taking into account $(\sim 1.3 \mathrm{deg})$ divergence of the incident beam) dependences of the angular distribution of the transmission coefficient through the test heterostructure in the absence of an electric field. The figure shows distinct oscillations in the transmission intensity, since the HES is a Fabry - Pérot interferometer (FPI). The comparison of the experimental curve with the model (5) allows us to determine not only the real, but also the imaginary part of the refractive index of the layers of the hydroelectric power station. The results for the test HES are presented in table 1. 


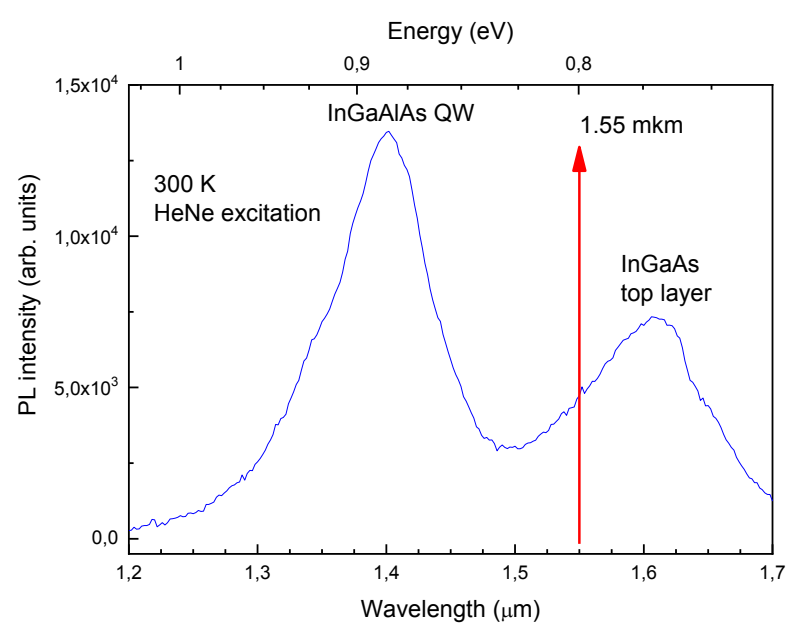

Fig. 3. Typical photoluminescence spectrum of the HES with InGaAlAs/InAlAs multiple quantum wells.

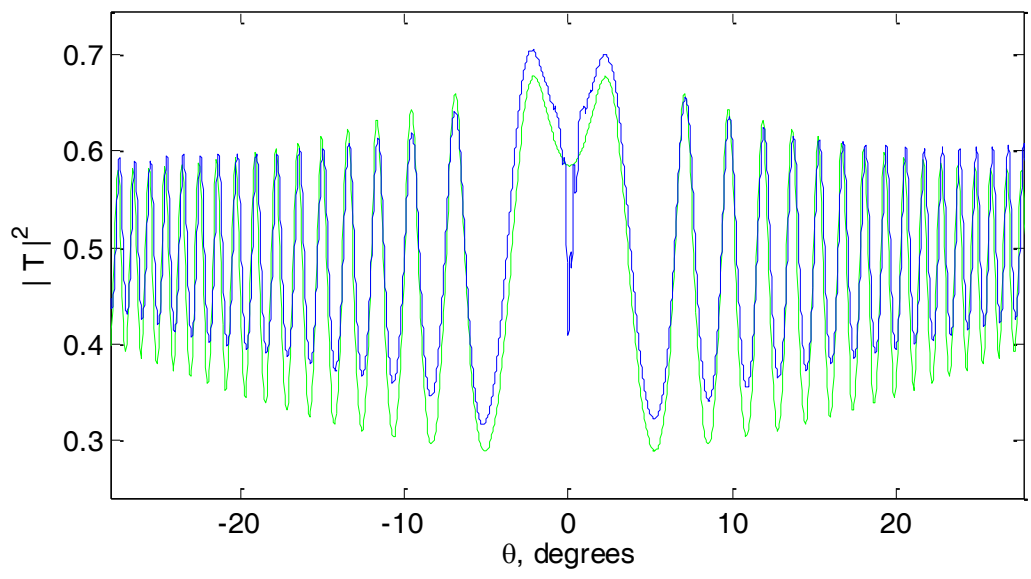

Fig. 4. Experimental (blue) and calculated (green) angle distributions of the coefficient of light transmission through the heterostructure.

Figure 5A shows a typical change in the angle dependence of the HES transmission coefficient upon voltage application. As one can see from Figure 5A, the application of an electric field to a HES leads not only to a change in signal intensity, but also a shift in the position of the extremum, which significantly increases the accuracy of measurements when choosing a fixed angle of the light incidence. According to the model (1-6), such a change in the interference pattern corresponds to a change in the refractive index of the active region of the HES by 0.01 The change in the transmittance of the HES from voltage at a fixed angle near one of the extrema as well as the corresponding calculated change in the effective refractive index of the active region of the HES are shown in Figure 5B. The required working length of the Mach-Zehnder interferometer can be easily estimated from this figure. In the Mach-Zehnder interferometer, the condition for complete signal suppression corresponds to the phase difference $\pi$ between its two channels: $\exp \left(i k_{0} \Delta n L\right)=\exp (i \pi)$. Then, the required working length of the Mach-Zehnder interferometer under a change of the refractive index in one $L_{I F}$ channel by $\Delta n$ is calculated by the formula: 


$$
L_{I F}=\lambda_{0} / 2 \Delta n
$$

For an operating point of $5 \mathrm{~V}$ and a RF voltage of $1 \mathrm{~V}$, the change in the refractive index of the active region of the HES is 0.0021. Thus, the sufficient length of the Mach-Zehnder interferometer at an InGaAlAs/InAlAs HES will be only $200 \mu \mathrm{m}$ when the modulator is in the push-pull mode.
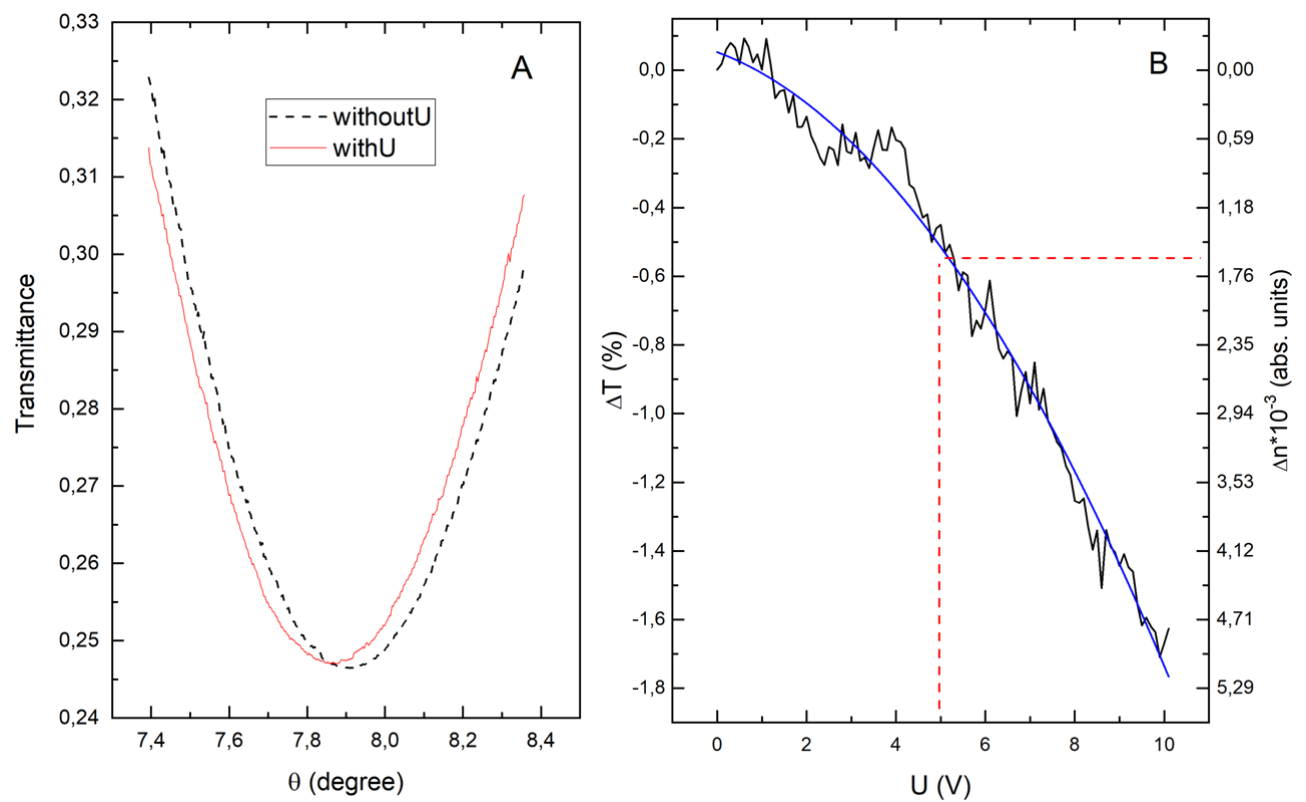

Fig. 5. (A) Angular distribution of the coefficient of transmission through a HES at $1.55 \mu \mathrm{m}$ wavelength without (black, dash-dotted line) and with a $8 \mathrm{~V}$ voltage (red, solid), (B) The dependence of the transmittance change of a HES on voltage (black ) and the corresponding simulated change in the effective refractive index of the active region of the HES (blue).

\section{Conclusion}

Thus, the value of the electric effect has been determined for heterostructures with the InGaAlAs / InAlsAs multiple quantum wells grown by the MBE method for an electrooptical modulator based on the Stark effect. It has been found that the effective index of the HES active region changes by $2 \cdot 10^{-3}$ at a voltage of $1 \mathrm{~V}$ and an operating point of $5 \mathrm{~V}$. At such an electro-optical effect level the sufficient length of the Mach-Zehnder interferometer does not exceed $200 \mu \mathrm{m}$.

This work was financially supported by the Russian Science Foundation (grant number 19-72-30023).

\section{References}

1. B. Jalali, Phys. Stat. Sol. (a) 205, 213-224 (2008)

2. Z. Zhang, N. Mettbach, C. Zawadzki, J. Wang, D. Schmidt, et al., IET Optoelectronics 5, 226-232 (2011)

3. W. Bogaerts, P. Dumon, D. van Thourhout, D. Taillaert, P. Jaenen, et al., IEEE J. Sel. Topics Quantum Electron. 12, 1394-1401 (2006) 
4. T. Ido et al. J. Lightwave Technol. 14, 2026-2034 (1996)

5. R. Nagarajan, M. Kato, J. Pleumeekers, P. Evans, S. Corzine, et al., IEEE J. Sel. Topics Quantum Electron. 16, 1113-1125 (2010)

6. A.V. Tsarev \& R.M. Taziev, Proceedings. 14th International Scientific-Technical Conference on Actual Problems of Electronic Instrument Engineering, APEIE, 301305 (2018)

7. D.V.Dmitriev et al, IOP Conf. Ser.: Mater. Sci. Eng. 475, 012022 (2019)

8. Integrated Optics. Edited by T.Tamir. Springer-Verlag, 344 pages (1975)

9. Scandonne F., Ballerini L., Nuovo Cemento 3, 81-91 (1946) 